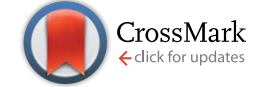

Cite this: RSC Adv., 2017, 7, 7015

Received 2nd November 2016 Accepted 12th January 2017

DOI: 10.1039/c6ra26213a

www.rsc.org/advances

\section{Highly sensitive and reliable SERS probes based on nanogap control of a $\mathrm{Au}-\mathrm{Ag}$ alloy on silica nanoparticles $\uparrow$}

\author{
Xuan-Hung Pham, $\dot{t}^{\mathrm{a}}$ Minwoo Lee,,$^{\mathrm{b}}$ Seongbo Shim, ${ }^{\mathrm{a}}$ Sinyoung Jeong, $\S^{\mathrm{b}}$ \\ Hyung-Mo Kim, ${ }^{a}$ Eunil Hahm, ${ }^{a}$ Sang Hun Lee, ${ }^{C}$ Yoon-Sik Lee, ${ }^{c}$ Dae Hong Jeong ${ }^{b}$ \\ and Bong-Hyun Jun*a
}

\begin{abstract}
We developed highly sensitive surface-enhanced Raman scattering (SERS) probes based on $\mathrm{SiO}_{2} @ \mathrm{Au} @ \mathrm{Ag}$ nanoparticles (NPs) using the Ag growth onto Au NP seeds method. The $\mathrm{SiO}_{2} @ A u @ A g$ NPs were synthesized by reducing Ag ions under mild conditions (ascorbic acid) and using the structure-directing agent polyvinylpyrrolidone (PVP). SERS activities of the NPs were tuned by adjusting $\mathrm{AgNO}_{3}$ concentration, resulting in the growth of the Ag shell on the surface of the Au NP seeds and the formation of narrow gaps between two Ag NPs on the surface of the probes. The NPs exhibited strong Raman signals originating from a highly enhanced $E$-field at the gaps. The $\mathrm{SiO}_{2} @ A$ AuaAg NPs exhibited a low limit of detection (LOD) value of $2.4 \mathrm{nM}$ for ATP, which proves that they are highly sensitive probes. Moreover, reproducible Raman signals of the $\mathrm{SiO}_{2} @ \mathrm{Au} a \mathrm{Ag}$ NPs toward ATP were obtained in batch-to-batch experiments which is very promising for potential use in on-site detection.
\end{abstract}

\section{Introduction}

Surface-enhanced Raman scattering (SERS) has attracted considerable interest for bioanalytical and imaging applications and in environmental monitoring as a non-destructive, ultrasensitive, and selective analytical technique. ${ }^{1-7}$ SERS enhancement occurs through both a short-range chemical mechanism (CM) and a long-range electromagnetic mechanism (EM) of metal substrates, such as Au or Ag. ${ }^{2}$ EM, which is a dominant SERS enhancement mechanism, is caused by localized surface plasmon resonance (LSPR) under excitation of light on or near the surface of metallic nanoparticles (NPs), resulting in an enhanced local electromagnetic field. ${ }^{8}$ Thus, the metal nanostructure and the SERS material components play important roles in generating the strong SERS signals. ${ }^{2,8}$ To improve SERS activity, nanomaterials of various sizes, structures, and components, such as nanospheres, nanoshells, nanorods, and multibranched NPs, have been

${ }^{a}$ Department of Bioscience and Biotechnology, Konkuk University, Seoul 143-701, Republic of Korea. E-mail: bjun@konkuk.ac.kr

${ }^{b}$ Department of Chemistry Education, Seoul National University, Seoul 151-742, Republic of Korea

${ }^{c}$ School of Chemical and Biological Engineering, Seoul National University, Seoul 151742, Republic of Korea

$\dagger$ Electronic supplementary information (ESI) available. See DOI: 10.1039/c6ra26213a

\$ These authors contributed equally to the work.

$\S$ Present address: Wellman Center for Photomedicine, Harvard Medical School, Massachusetts General Hospital, 149 13th Street, Charlestown, Massachusetts 02129, USA. developed and used as SERS probes., ${ }^{\mathbf{4}-12}$ However, SERS signals from single NPs might not always have sufficient sensitivity. ${ }^{2,4}$

Bimetallic $\mathrm{Au}$ and $\mathrm{Ag}$ NPs have attracted extensive interest in SERS because the LSPR of Au-Ag alloy NPs enhances EM, which plays an important role in SERS. Au-Ag alloy NPs offer several advantages, including enhanced SERS signal broad band absorption of light due to bimetallic materials, and dynamic modulation of electronic properties without altering overall size. ${ }^{13}$ Moreover, they possess the long-term stability and biocompatibility due to $\mathrm{Au}$ and give extraordinary enhancement of Raman intensities due to $\mathrm{Ag}$ nanostructure..$^{13}$ Especially, using the seed mediated growth method, by adjusting the Ag source amount, the growth of Ag shell thickness on the Au seeds can be reliably controlled. ${ }^{\mathbf{1 1 , 1 2 , 1 4 - 1 6}}$ A SERS probe based on aggregation of metal ( $\mathrm{Au}$ and/or $\mathrm{Ag}$ ) NP clusters has been fabricated for enhanced SERS signals. Signals from a NP-cluster probe are dramatically enhanced up to $10^{10}$ compared to that of a single NP-based SERS probe because the presence of the junction between two NPs, which are commonly called "hot spots", arouse LSPR on or near the aggregated NPs., ${ }^{2,17}$ Several strategies have been used to obtain aggregated NP clusters and they are cataloged into two; one is to induce nanoaggregates without template by using salt $^{18}$ or by Raman reporters. ${ }^{19}$ However, precise and reproducible control of the aggregated NP clusters has been difficult due to the formation of heterogeneity in their local structure. ${ }^{\mathbf{1 9 2 0}}$ The other is to generate nanoaggregate structures using a template, such as silica particles or polymer beads, to deposit much smaller Au or Ag NPs onto their surfaces. ${ }^{20-30}$ In this strategy, the silica NPs are widely used as 
a template for metal NPs as they are inert, and their sizes are easily controllable. So far, although the assembly of Au and/or $\mathrm{Ag}$ NPs onto silica NPs has been reported by several groups, ${ }^{4,15,23-27,31-39}$ the density of the Au and/or Ag NPs and the gaps between two NPs which can provide a stronger LSPR property are not studied well. ${ }^{40}$

In this study, we report an easy method to prepare nanogaps containing $\mathrm{Au}-\mathrm{Ag}$ NPs on silica NPs $\left(\mathrm{SiO}_{2} @ \mathrm{Au} @ \mathrm{Au} \mathrm{NPs}\right)$ for the sensitive detection of chemicals as strong and reliable SERS probe. The $\mathrm{SiO}_{2}$ @Au@Ag NPs were synthesized by using the Au seed mediated Ag growth method. This approach allowed us to perform the controlled synthesis of Ag shell on Au NPs surfaces of silica NP. The variation of SERS activities of the $\mathrm{SiO}_{2} @ \mathrm{Au} @ \mathrm{Ag}$ NPs in relation to nanogaps in the presence of 4-aminobenzophenol (4-ATP) was obtained. The variation of SERS spectra of the $\mathrm{SiO}_{2} @ A u @ A g$ NPs with various ATP to demonstrate their potential as sensitive chemical detection probes. Furthermore, we also confirmed a relationship between the SERS signal and the distance of Ag NPs on the surface of silica NPs by simulation.

\section{Results and discussion}

\subsection{Preparation of $\mathrm{SiO}_{2} @ \mathrm{Au} @ \mathrm{Ag} \mathrm{NPs}$}

The procedures to synthesize the $\mathrm{SiO}_{2} @ \mathrm{Au} @ \mathrm{Ag}$ NPs are illustrated in Fig. 1. Amine-functionalized silica NPs ( $c a .150 \mathrm{~nm}$ in diameter) were used as a dielectric core. Colloidal Au NPs (2-3 nm) were prepared by reducing gold(III) chloride with tetrakis(hydroxymethyl)-phosphonium chloride (THPC), according to the method reported by Duff et al. with slight modifications. ${ }^{41}$ The Au NPs were incubated with aminated silica NPs for $12 \mathrm{~h}$ by gently shaking at room temperature (Fig. S1 $\dagger$ ). The surfaces of the aminated silica NPs were covered with $\sim 2300$ unit of Au NPs. The amine functional group plays a crucial role in attaching Au NPs through strong electrostatic

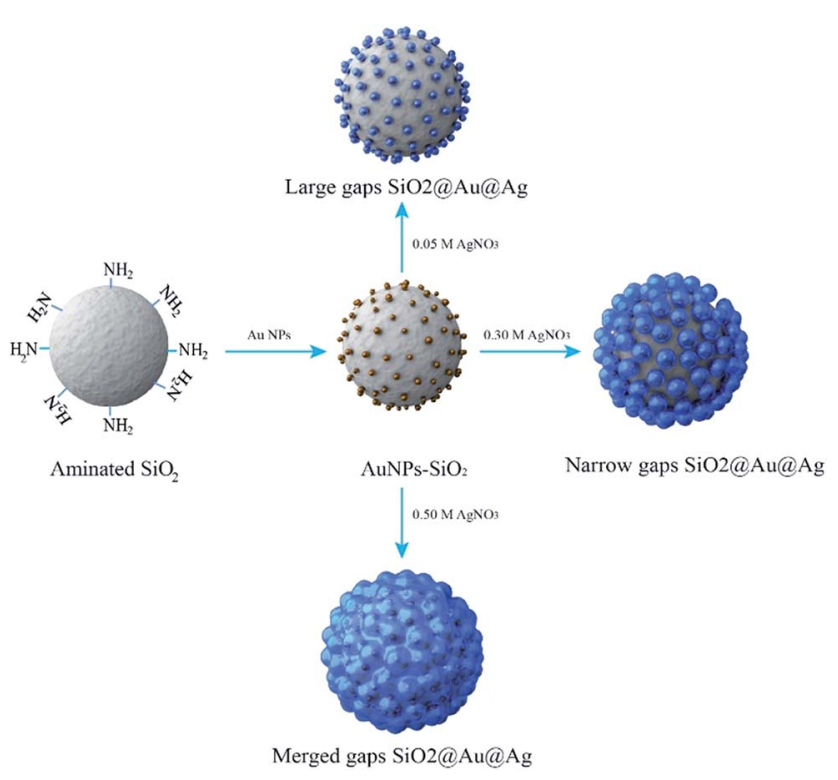

Fig. 1 Typical preparation procedure of the $\mathrm{SiO}_{2}(\mathrm{aAu} @ \mathrm{Ag}$ NPs. attractions and thus, facilitating Ag shell growth on Au NPs on the silica surface. The Ag shell was deposited selectively on Au NPs by reducing a silver precursor $\left(\mathrm{AgNO}_{3}\right)$ on the silica surface in the presence of ascorbic acid and polyvinylpyrrolidone (PVP). PVP is a stabilizer and a structure-directing agent that played a crucial role in covering the Ag shell on the Au NPs on the silica surface under the mild reducing conditions (Fig. S2 $\dagger$ ). PVP adsorbed onto Au NPs facilitated the reduction of $\mathrm{Ag}$ ions onto the $\mathrm{SiO}_{2} @ \mathrm{Au}$ NPs. ${ }^{42,43}$

Next, we determined the effect of the size of the Ag NPs and their gaps on the $\mathrm{SiO}_{2} @ \mathrm{Au}$ NPs in relation to SERS signal by adjusting the concentration of $\mathrm{AgNO}_{3}$ in the solution. The quantity of the $\mathrm{SiO}_{2} @ \mathrm{Au} \mathrm{NPs}$ was fixed at $0.2 \mathrm{mg}$ and the $\mathrm{AgNO}_{3}$ concentration was varied from 50 to $500 \mu \mathrm{M}$. A representative transmission electron microscopic (TEM) image is shown in Fig. 2A. When the $\mathrm{AgNO}_{3}$ was increased from 50 to $300 \mu \mathrm{M}$, the size of the Au@Ag alloy NPs became larger on the surface of the $\mathrm{SiO}_{2} @ \mathrm{Au}$ NPs as seen in Fig. 2B(a). In particular, the alloy diameter measured from high resolution (HR)-TEM and analyzed by Image J software, increased to $14.1 \pm 3.4(50 \mu \mathrm{M})$, $18.4 \pm 4.4(100 \mu \mathrm{M}), 31.4 \pm 8.4(200 \mu \mathrm{M})$ and $45.7 \pm 17.4 \mathrm{~nm}$ $(300 \mu \mathrm{M})$. To provide more information of $\mathrm{Ag}$ coating, the thickness of the Ag shell was calculated by subtracting the radius of the Au NPs from the radius of the Au@Ag NPs. Here, we assumed that the Au@Ag alloy NPs were spheres, each Au@Ag NP contained one Au NP and the Au NPs maintained their size. The thickness of the Ag shell was determined to be 7.0 \pm 1.7 to $22.9 \pm 8.7 \mathrm{~nm}$ when $\mathrm{AgNO}_{3}$ concentration increase from $50 \mu \mathrm{M}$ to $300 \mu \mathrm{M}$ in Fig. $2 \mathrm{~B}$ (c). In contrast, the nanogap between Ag NPs on the surface of the $\mathrm{SiO}_{2} @ \mathrm{Au}$ NPs decreased with increasing $\mathrm{AgNO}_{3}$ concentration from 50 to $300 \mu \mathrm{M}$. The gaps between two Au@Ag alloy NPs was decreased from $7.4 \pm$ 6.1 to $1 \pm 1.6 \mathrm{~nm}$ and the $\mathrm{Ag}$ shell was partly merged at $>200 \mathrm{mM}$ $\mathrm{AgNO}_{3}$, leading the decrement of the "hot spot" on the surface of the $\mathrm{SiO}_{2} @ A u @ A g$ NPs in Fig. 2B(d). However, the size of Ag NPs on the $\mathrm{SiO}_{2} @ A u$ NPs surfaces became larger and irregularly shaped at higher concentrations of $\mathrm{AgNO}_{3}(>300 \mu \mathrm{M})$.

The UV-Vis absorption spectra of the $\mathrm{SiO}_{2} @ \mathrm{Au} @ \mathrm{Ag}$ NPs shown in Fig. 2B(d) were well matched with the TEM images. $\mathrm{SiO}_{2} @ \mathrm{Au}$ NPs did not show the typical UV peak of Au NPs at 500-520 nm because of low absorbance of the 2-3 nm sized Au NPs. ${ }^{41}$ After depositing the $\mathrm{Ag}$ onto the $\mathrm{SiO}_{2} @ \mathrm{Au}$ NPs at $50 \mu \mathrm{M}$ of $\mathrm{AgNO}_{3}$, a single plasmonic absorption band at $\sim 410 \mathrm{~nm}$ was observed which is ascribed to the unique optical property of separated Ag NPs on the $\mathrm{SiO}_{2} @ A u$ surfaces. However, this band was red-shifted to $430 \mathrm{~nm}$, and the wavelength broadened from 400 to $800 \mathrm{~nm}$ as $\mathrm{AgNO}_{3}$ concentration was increased from 50 to $300 \mu \mathrm{M}$, indicating that $\mathrm{Ag}$ shell on the surfaces of Au NPs on the silica $\mathrm{NPs}^{44}$ created hot-spot structures on the $\mathrm{SiO}_{2} @ \mathrm{Au} @ \mathrm{Ag}$ NPs. This absorption may be due to the diversity in the size of the Ag clusters, which produces a continuous spectrum of resonant multimode. ${ }^{\mathbf{4 4 , 4 5}}$ However, when $\mathrm{AgNO}_{3}$ concentration increased to more than $400 \mu \mathrm{M}$, absorbance increased dramatically compared to that of the $\mathrm{SiO}_{2} @ \mathrm{Au} @ \mathrm{Ag}$ NPs synthesized with $300 \mu \mathrm{M}$ of $\mathrm{AgNO}_{3}$. This might be due to the partial merging of Ag NPs to larger Ag NPs on the surface of the $\mathrm{SiO}_{2}$ @Au@Ag NPs as shown in Fig. 2B. Therefore, the 

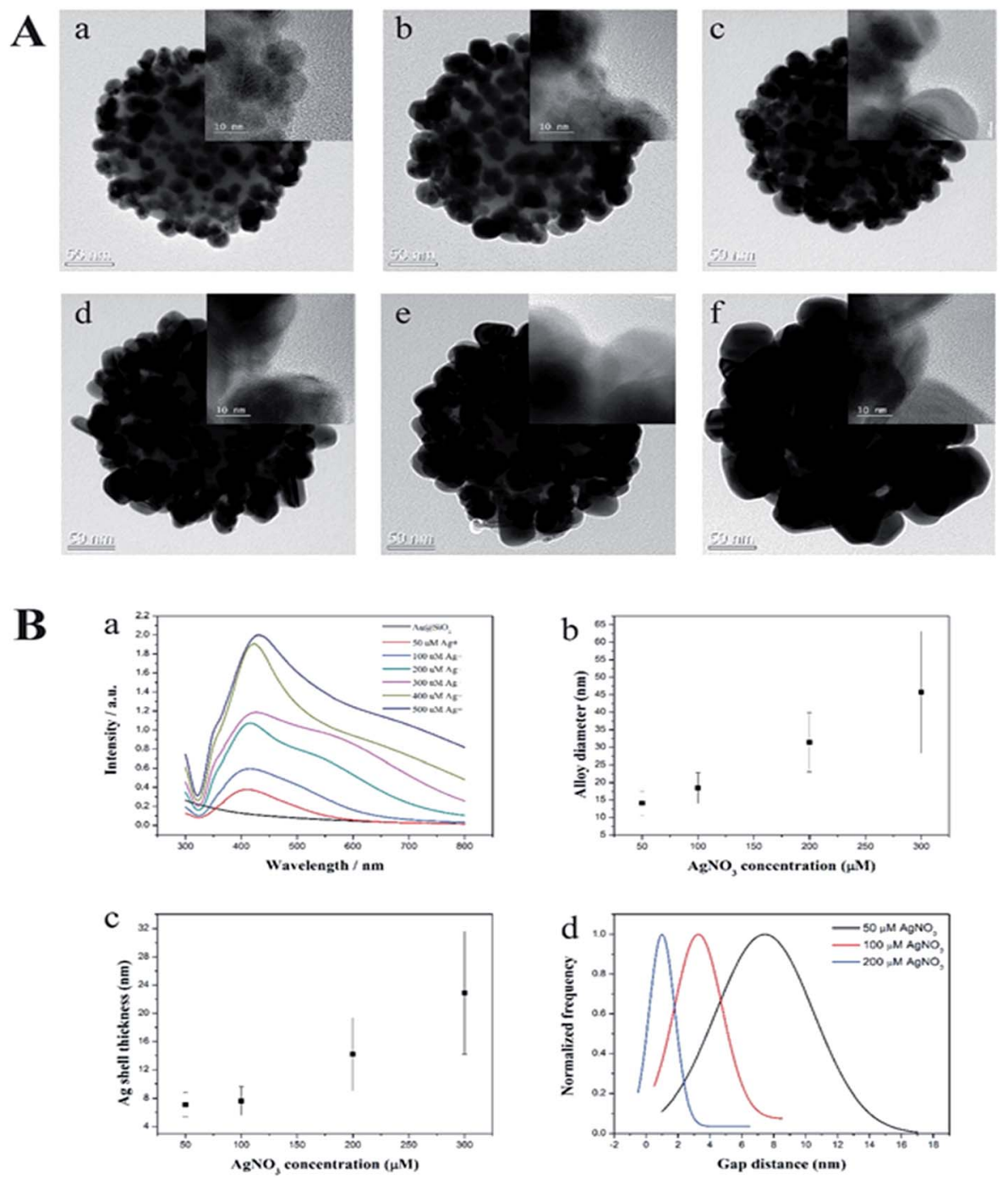

Fig. 2 (A) Transmission electron micrographs of the $\mathrm{SiO}_{2} @ \mathrm{aAu} @ \mathrm{Ag}$ NPs synthesized with (a) $50 \mu \mathrm{M} \mathrm{AgNO}_{3} ;$ (b) $100 \mu \mathrm{M} \mathrm{AgNO}_{3}$; (c) $200 \mu \mathrm{M} \mathrm{AgNO}$; (d) $300 \mu \mathrm{M} \mathrm{AgNO}_{3}$; (e) $400 \mu \mathrm{M} \mathrm{AgNO}_{3}$, and (f) $500 \mu \mathrm{M} \mathrm{AgNO}_{3}$. (B) (a) UV-vis absorption spectra, (b) Au@Au diameter, (c) Ag shell thickness and (d) gap distance of the $\mathrm{SiO}_{2}$ (aAu@Ag NPs.

$\mathrm{SiO}_{2} @ A u @ A g$ NPs prepared from $2.5 \mathrm{~nm}$ Au NPs exhibited a longitudinal UV-vis spectrum with a coupling of inner Au NPs core and outer Ag shell that is similar to the absorbance pattern of individual Ag NPs of large size (100-150 nm). ${ }^{44}$ Elemental mapping with energy dispersive X-ray spectroscopy (EDX) was applied to confirm the presence of the $\mathrm{Ag}$ on the $\mathrm{SiO}_{2} @ \mathrm{Au} @ \mathrm{Ag}$ prepared with $300 \mu \mathrm{M} \mathrm{AgNO}$ in Fig. S3. $\uparrow$ The atomic element compositional analysis of the $\mathrm{SiO}_{2} @ \mathrm{Au} @ \mathrm{Ag}$ NPs confirmed $28.3 \% \mathrm{Si}, 42.5 \% \mathrm{O}, 0.8 \% \mathrm{Au}$, and $28.4 \% \mathrm{Ag}$ (Fig. S4†). Au atoms were observed on the surface of the core silica NPs, whereas high density $\mathrm{Ag}$ atoms were detected at the outer layer of the $\mathrm{SiO}_{2} @ A u @ A g$, indicating that a thick Ag shell covered the $\mathrm{Au}$ NPs on silica NPs.

\subsection{SERS activity of the $\mathrm{SiO}_{2} @ A u @ A g$ NPs}

The SERS-activities of these materials were investigated using 4aminothiophenol (ATP) as a Raman chemical. To demonstrate the advantage of the $\mathrm{SiO}_{2} @ A u @ A g$ NPs, we synthesized $\mathrm{SiO}_{2}$ @Ag NPs according to the method reported by Kang et l. $^{25}$ Both the $\mathrm{SiO}_{2} @ A g$ and $\mathrm{SiO}_{2} @ A u @ A g$ NPs were incubated with ATP to immobilize the Raman chemical on the surface of the probes. The Raman signals were recorded using a micro Raman system after dropping and air-drying the solution $(10 \mu \mathrm{L})$ onto a glass substrate and the SERS measurement results are shown in Fig. 3A. The SERS signal of the $\mathrm{SiO}_{2} @ A$ A NPs was weak and unclear because of low optical absorption of the Au NPs compared to that of the Ag NPs. ${ }^{46}$ Typical ATP bands were obtained clearly for both the $\mathrm{SiO}_{2} @ A g$ and $\mathrm{SiO}_{2} @ A u @ A g$ due to the electromagnetic enhancement of the decorated Ag NPs. Dominant and distinct ATP peaks were at 1142 and $1432 \mathrm{~cm}^{-1}$ were assigned to $\mathrm{C}-\mathrm{H}$ in-plane and out-of-plane bending vibrations while the peak at $1390 \mathrm{~cm}^{-1}$ was due to $N$-phenyl stretching. The peaks at 1575 and $1078 \mathrm{~cm}^{-1}$ were attributed to ring $\mathrm{C}-\mathrm{C}$ and C-S stretching, respectively. ${ }^{47}$ Interestingly, the SERS signal of the $\mathrm{SiO}_{2} @ A u @ A g$ NPs at $1078 \mathrm{~cm}^{-1}$ was $\sim 3185 \mathrm{cps}$ with $10 \mathrm{mM}$ 

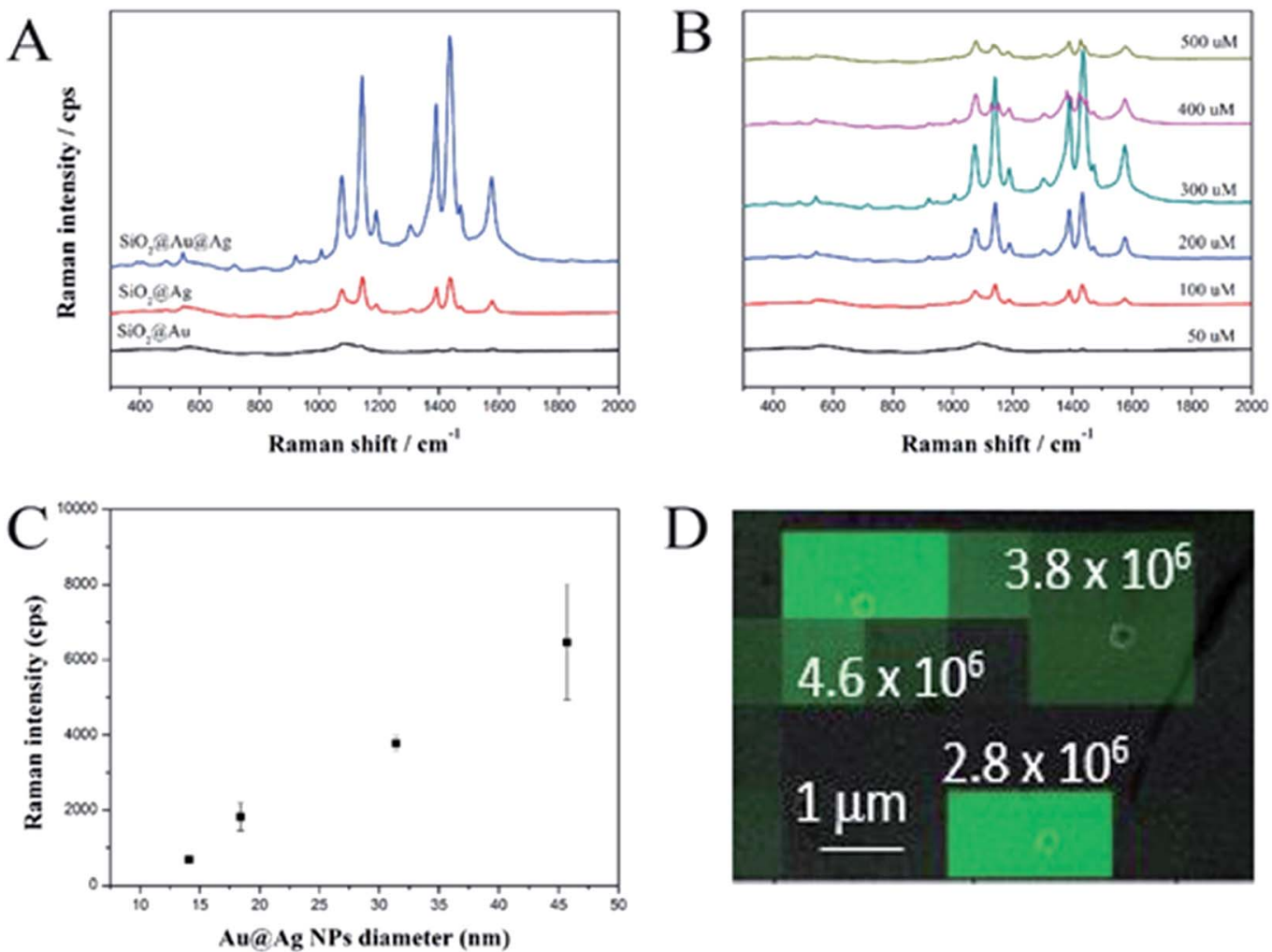

Fig. 3 (A) Comparison of SERS spectra of $\mathrm{SiO}_{2} @ \mathrm{AAu}, \mathrm{SiO}_{2} @ \mathrm{Ag}$, and $\mathrm{SiO}_{2} @ \mathrm{QAu} @ \mathrm{Ag} \mathrm{NPs}$ at a $0.2 \mathrm{mg} \mathrm{mL}^{-1}$ concentration of $4-\mathrm{ATP}$. (B) Effect of $\mathrm{AgNO}_{3}$ concentration and (C) Au@Ag diameter on SERS signals of $\mathrm{SiO}_{2}$ (aAu@Ag toward ATP. SERS spectra of 10 mM ATP were obtained using a $532 \mathrm{~nm}$ laser with $10 \mathrm{~mW}$ power for $5 \mathrm{~s}$. (D) SERS intensity map was overlaid with its corresponded SEM image. Distribution of enhancement factor for the $1099 \mathrm{~cm}^{-1}$ Raman band of the $\mathrm{SiO}_{2} @ A$ Au@Ag NPs bearing 4-FBT. Based on the calculation from 75 single SiO ${ }_{2} @ A$ AuAg NPs, the EF was $4.2 \times 10^{6}$.

ATP, which was 3.5-fold stronger than the SERS signal of $\mathrm{SiO}_{2} @ \mathrm{Ag}$ NPs even though $\mathrm{SiO}_{2} @ A g$ NPs possessed many nanogaps among Ag NPs as shown in Fig. S5. $\dagger$ Similarly, these values were 3.8-, 4.0-, 4.5-, and 3.5-fold stronger at 1142, 1390, 1432 , and $1575 \mathrm{~cm}^{-1}$, respectively. This result demonstrates the importance of pre-depositing Au NPs onto the $\mathrm{SiO}_{2}$ surface to ensure the growth of $\mathrm{Ag}$ shell and amplify the SERS signal.

In addition, the effect of $\mathrm{AgNO}_{3}$ concentration on the SERS signals of the $\mathrm{SiO}_{2} @ A u @ A g$ NPs with 10 mM ATP was investigated (Fig. 3B). Signal intensity was proportional to $\mathrm{AgNO}_{3}$ concentration from 50 to $300 \mu \mathrm{M}$ and was the highest at $300 \mu \mathrm{M}$, such as $\sim 3185 \mathrm{cps}\left(1078 \mathrm{~cm}^{-1}\right) ; 3297 \mathrm{cps}\left(1142 \mathrm{~cm}^{-1}\right) ; 4320 \mathrm{cps}$ $\left(1142 \mathrm{~cm}^{-1}\right)$; $4515 \mathrm{cps}\left(1142 \mathrm{~cm}^{-1}\right)$; and $2677 \mathrm{cps}\left(1575 \mathrm{~cm}^{-1}\right)$. These results are consistent with the TEM images shown in Fig. 2A. The gaps between Ag NPs was about $7.4 \pm 6.1 \mathrm{~nm}$ at 50 $\mu \mathrm{M} \mathrm{AgNO}$, which did not strongly enhance the signals of ATP molecules on the surfaces of the $\mathrm{SiO}_{2} @ A u @ A g$ NPs. When the $\mathrm{AgNO}_{3}$ concentration was increased to $300 \mu \mathrm{M}$, the nanogaps became $<1.0 \mathrm{~nm}$, which can create "hot-spots", and induce a strong enhancement of the electromagnetic field surrounding the $\mathrm{SiO}_{2} @ A u @ A g$ NPs. However, the SERS signals of the $\mathrm{SiO}_{2} @ A u @ A g$ NPs synthesized at higher concentrations of $\mathrm{AgNO}_{3}(400$ and $500 \mu \mathrm{M})$ were decreased dramatically because there was no nanogap in the $\mathrm{Ag}$ shell. Moreover, the shape of $\mathrm{Ag}$ NPs became irregular. Therefore, the optimal $\mathrm{AgNO}_{3}$ concentration was $300 \mu \mathrm{M}$.
Additionally, we measured SERS intensity of a single $\mathrm{SiO}_{2} @-$ Au@Ag NPs labelled with 4-fluoro thiophenol (4-FBT) to evaluate the enhancement factor and reproducibility of SERS signal. The $\mathrm{SiO}_{2} @ A u @ A g$ NPs solution (0.02 mg mL ${ }^{-1}$ in EtOH) was dropcast on a patterned slide glass to easily monitor the $\mathrm{SiO}_{2}$ @Au@Ag NPs using scanning electron microscopy (SEM). After mapping the SERS signals of the $\mathrm{SiO}_{2} @ A u @ A g$ NPs, the SERS intensity maps were overlaid on the corresponding SEM image to measure the intensity of the SERS signal from a single $\mathrm{SiO}_{2}$ @Au@Ag NPs in the entire SERS intensity map as mentioned in Fig. 3. The signal of 4-FBT peak at $1099 \mathrm{~cm}^{-1}$ was used to record the SERS intensity and calculate the enhancement factor (EF) (see Fig. S7, ESI $\dagger$ ). As results, the area of strong signals on the SERS intensity map corresponded to the position of the single $\mathrm{SiO}_{2} @ A u @ A g$ NPs in the SEM image, indicating that the SERS signal from a single particle was strong enough to be detectable. Additionally, we calculated the SERS EFs for 75 single $\mathrm{SiO}_{2}$ @Au@Ag NPs and their mean SERS EF value was $4.2 \times 10^{6}$.

Finally, we used the $\mathrm{SiO}_{2} @ \mathrm{Au} @ A \mathrm{Ag}$ NPs as active SERS probes for label-free detection of ATP in a model study. The $\mathrm{SiO}_{2} @-$ Au@Ag NPs were mixed with various concentrations of ATP in ethanol solution. Fig. 4A shows the strong SERS signals of ATP at each concentration $\left(10^{-2}\right.$ to $\left.10^{-8} \mathrm{M}\right)$, confirming that the $\mathrm{SiO}_{2} @ A u @ A g$ NPs are an active SERS substrate to detect ATP.

Specifically, the intensities of the Raman bands at 1078, 1142, 1390, 1432, and $1575 \mathrm{~cm}^{-1}$ increased as ATP concentration 

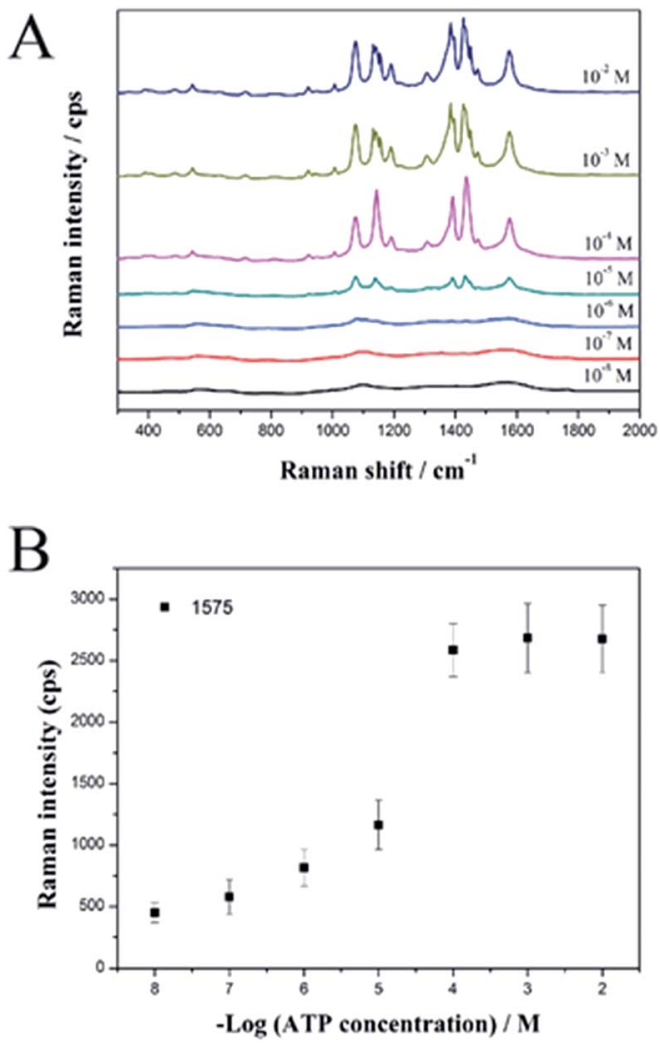

Fig. 4 (A) SERS spectra and (B) calibration curves for the $\mathrm{SiO}_{2}$ (a) Au@Ag NPs at different ATP concentration of $10^{-8}$ to $10^{-2} \mathrm{M}$. SERS spectra of $10 \mathrm{mM}$ ATP at $1575 \mathrm{~cm}^{-1}$ were obtained using a $532 \mathrm{~nm}$ laser with 10 $\mathrm{mW}$ power for $5 \mathrm{~s}$ with the $\times 10$ objective lens $(0.25 \mathrm{NA})$.

increased. Fig. 4B shows the linear correlation between peak intensity and ATP concentration. In support of the sensitive nature of the NP-based label-free detection, we calculated the theoretical LOD for the $\mathrm{SiO}_{2} @ A u @ A g$ system to detect ATP at the $1575 \mathrm{~cm}^{-1}$ peak, which is based on the standard deviation of the response and the slope of the calibration curve at levels approximating the LOD according to the formula: $\mathrm{LOD}=3.3(\mathrm{SD} / S)$. The theoretical LOD of the $\mathrm{SiO}_{2} @ A u @ A g$ NPs was $2.4 \mathrm{nM}$, which is much lower than the $\mathrm{SiO}_{2} @ \mathrm{Ag}$ NPs with a LOD of $6 \mu \mathrm{M}^{27}$

We also performed batch-to-batch reproducibility experiments. The SERS signals of ATP on $\mathrm{SiO}_{2} @ A u @ A g$ NPs obtained from three different batches were calculated at $1575 \mathrm{~cm}^{-1}$. As shown in Fig S8, $\uparrow$ the signals were only varied from 2500-3000 cps ( $\sim 14.2 \%$ deviation) with a high reproducible Raman signals of the $\mathrm{SiO}_{2} @ \mathrm{Au} @ \mathrm{Ag}$ NPs.

\subsection{Simulation of theoretical Au NPs $E$-field distributions on the Au@Ag NPs dimer for identifying SERS enhancement}

To support the dependency of SERS enhancement on the quantity of $\mathrm{Ag}$ in the $\mathrm{SiO}_{2} @ \mathrm{Au} @ \mathrm{Ag}$ NPs with different nanogaps, we carried out a discrete dipole approximation (DDA) simulation for the theoretical $E$-field calculation of $\mathrm{Ag}, \mathrm{Au}$ and $\mathrm{Au} @ A g$ NPs dimer in Fig. 5 and S9. $\dagger$ The core diameter of $\mathrm{Au}$ NPs of Au@Ag NPs dimer was set to $3 \mathrm{~nm}$ and Ag shell thickness of the Au@Ag NPs dimer was set to 2.5-10 nm. The Au@Ag NPs
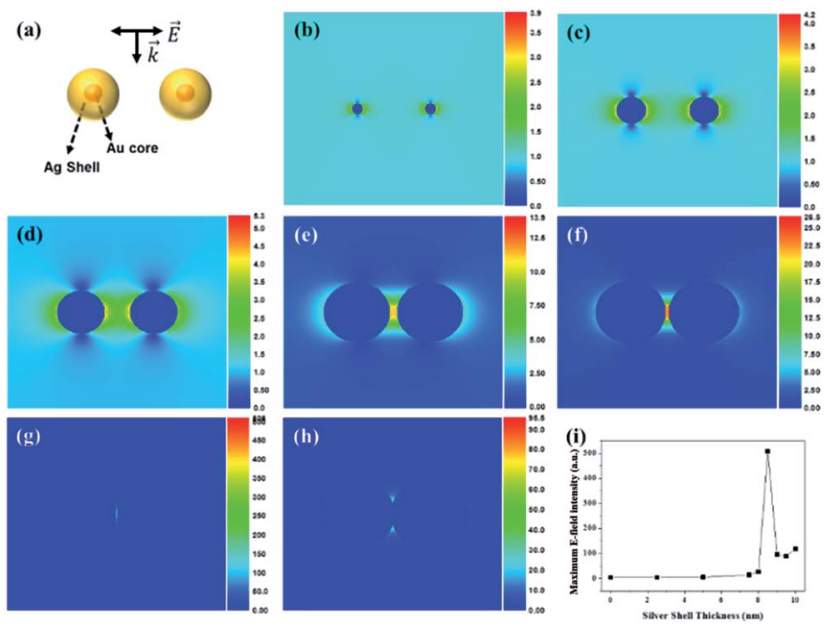

Fig. 5 (a) Calculated structure of Au NPs and the Au@Ag NPs dimer, theoretical $E$-field distribution of (b) the Au NPs dimer (17.5 nm gap) and the Au@Ag NPs dimer with (c) $2.5 \mathrm{~nm}$ Ag shell (12.5 nm gap), (d) $5 \mathrm{~nm}$ (7.5 nm gap), (e) $7.5 \mathrm{~nm}$ (2.5 nm gap), (f) $8 \mathrm{~nm}$ (1.5 nm gap), (g) $8.5 \mathrm{~nm}(0.5 \mathrm{~nm}$ gap), (h) $9 \mathrm{~nm}$ (merging), and (i) maximum $E$-field intensity of Au NPs and the Au (aAg NPs dimer as Ag shell thickness was increased from 2.5 to $10 \mathrm{~nm}$.

dimer was merged after Ag shell thickness was $9 \mathrm{~nm}$. The theoretical $E$-field distribution of each structure and the maximum E-field intensity of each structure are shown in Fig. 5. As the Ag shell thickness increased gradually to $8.5 \mathrm{~nm}$, the interparticle gap of the Au@Ag NPs dimer became smaller, and the theoretical $E$-field intensity increased because of plasmon coupling between the Au@Ag NPs. ${ }^{48}$ On the other hand, as the Ag shell thickness became more than 9 nm, the Au@Ag NPs dimers merged and the theoretical $E$-field intensity decreased.

For identification of the contribution of $\mathrm{Au}$ seeds in $\mathrm{SiO}_{2} @ A u @ A g$ NPs, theoretical maximum $E$-field intensity of $\mathrm{Au} @ A g$ NPs was compared with those of Ag and Au NPs dimer as shown in Fig S9. $\uparrow$ The Au@Ag NPs dimer and Ag NPs dimer exhibited stronger intensity than Au NPs dimer as the particle distance become shorter, but the maximum $E$-field intensity of $\mathrm{Au} @ A g$ NPs dimer was not so different compared with that of Ag NPs dimer. However, in terms of particle size controlling, $\mathrm{Au}-\mathrm{Ag}$ alloy NPs on $\mathrm{SiO}_{2}$ surface was more controllable than Ag NPs on $\mathrm{SiO}_{2}$ surface.

Thus, the DDA simulation data supported well the SERS enhancement depending on the structural morphology changes of $\mathrm{SiO}_{2} @ \mathrm{Au} @ \mathrm{Ag} \mathrm{NPs}$ with increasing $\mathrm{AgNO}_{3}$ concentration.

\section{Experimental}

\subsection{Materials}

Tetraethylorthosilicate (TEOS), 3-mercaptopropyl trimethoxysilane (MPTS), 3-aminopropyltriethoxysilane (APTS), silver nitrate $\left(\mathrm{AgNO}_{3}\right)$, tetrakis(hydroxymethyl)phosphonium chloride (THPC), gold(III) chloride trihydrate $\left(\mathrm{HAuCl}_{4}\right)$, ascorbic acid (AA), 4-aminobenzenethiol (4-ATP), polyvinyl pyrrolidone (PVP), thiram were purchased from Sigma-Aldrich (St. Louis, MO, USA) and used without further purification. Ethyl alcohol (EtOH), 
and aqueous ammonium hydroxide $\left(\mathrm{NH}_{4} \mathrm{OH}, 27 \%\right)$ was purchased from Daejung (Siheung, Korea).

\subsection{Preparation of aminated silica nanoparticle (NP) templates}

Approximately $150 \mathrm{~nm}$ sized silica NPs were prepared using the Stöber method. ${ }^{49}$ Absolute EtOH $(40 \mathrm{~mL}), \mathrm{NH}_{4} \mathrm{OH}(3 \mathrm{~mL})$, and $1.6 \mathrm{~mL}$ TEOS $(1.6 \mathrm{~mL})$ were added to a round bottle. The solution was stirred vigorously at $700 \mathrm{rpm}$ for $20 \mathrm{~h}$ at $25{ }^{\circ} \mathrm{C}$. The silica NPs were centrifuged at $8500 \mathrm{rpm}$ for $15 \mathrm{~min}$ and washed several times with EtOH. The silica NPs $\left(50 \mathrm{mg} \mathrm{mL}^{-1}, 4 \mathrm{~mL}\right)$ were dispersed in $4 \mathrm{~mL}$ absolute EtOH, and $250 \mu \mathrm{L}$ APTS and 40 $\mu \mathrm{L} \mathrm{NH}_{4} \mathrm{OH}$ were added to the colloidal solution to obtain aminated silica NPs. The mixture was stirred vigorously for $6 \mathrm{~h}$ at $25{ }^{\circ} \mathrm{C}$, followed by stirring at $70{ }^{\circ} \mathrm{C}$ for $1 \mathrm{~h}$. The aminated silica NPs were obtained after centrifugation, and washed several times with EtOH to remove excess reagent.

\subsection{Preparation of the $\mathrm{SiO}_{2} @ A u$ NPs}

The colloidal Au NPs were prepared by reducing gold(III) chloride, using THPC (tetrakis(hydroxymethyl)-phosphonium chloride) as a reducing agent. The THPC capped Au NPs were prepared by a method reported of Duff et $a .^{41}$ The solution was stored at $4{ }^{\circ} \mathrm{C}$ for 3 days before use. Au NPs $(1 \mathrm{mM}, 10 \mathrm{~mL})$ and the aminated $\mathrm{SiO}_{2}$ solution $\left(1 \mathrm{mg} \mathrm{mL} \mathrm{mL}^{-1}, 1 \mathrm{~mL}\right)$ was mixed, sonicated for $30 \mathrm{~min}$, and incubated in a shaker overnight. ${ }^{33} \mathrm{Au}$ NPs-embedded silica NPs were obtained by centrifugation and washed several times with EtOH to remove unbound Au NPs. $\mathrm{SiO}_{2} @ \mathrm{Au} \mathrm{NPs}$ were redispersed into absolute EtOH to obtain $1 \mathrm{mg} \mathrm{mL}^{-1} \mathrm{SiO}_{2} @$ Au NPs solution.

\subsection{Preparation of the $\mathrm{SiO}_{2} @ \mathrm{Au} @ \mathrm{Ag} \mathrm{NPs}$}

$\mathrm{Au}-\mathrm{Ag}$ core-shell NPs were prepared in an aqueous medium by depositing Ag source with ascorbic acid on gold NPs in the presence of PVP. Briefly, $0.2 \mathrm{mg}$ of $\mathrm{SiO}_{2} @ \mathrm{Au}$ NPs were dispersed into $9.8 \mathrm{~mL}$ of water containing $10 \mathrm{mg}$ PVP and kept still for $30 \mathrm{~min}$. Silver nitrate $(10 \mathrm{mM}, 20 \mu \mathrm{L})$ was added to the solution, followed by $10 \mathrm{mM}$ ascorbic acid $(20 \mu \mathrm{L})$. This solution was incubated for $15 \mathrm{~min}$ to reduce $\mathrm{Ag}^{+}$to $\mathrm{Ag}$ metal. These steps were repeated to obtain the desired Ag concentrations (50, 100, 200, 300, 400, and $500 \mu \mathrm{M}$ of $\mathrm{AgNO}_{3}$ ). The $\mathrm{SiO}_{2} @ \mathrm{Au} @ \mathrm{Ag} \mathrm{NPs}$ were obtained by centrifugation at $8500 \mathrm{rpm}$ for $15 \mathrm{~min}$ and washed several times with EtOH to remove excess reagent. The $\mathrm{SiO}_{2} @ A u @ A g$ NPs were redispersed in $1.0 \mathrm{~mL}$ absolute EtOH to obtain $0.2 \mathrm{mg} \mathrm{mL} \mathrm{mL}^{-1} \mathrm{SiO}_{2}$ @Au@Ag NPs solution (based on $\mathrm{SiO}_{2}$ ).

\subsection{Incorporating Raman reporter materials into the $\mathrm{SiO}_{2} @ A u @ A g$ NPs}

A 4-ATP solution $(1 \mathrm{~mL}, 10 \mathrm{mM}$ in EtOH) was added to the $\mathrm{SiO}_{2} @ A u @ A g$ NPs $(0.2 \mathrm{mg})$, and the suspension was stirred vigorously for $2 \mathrm{~h}$ at $25{ }^{\circ} \mathrm{C}$. The colloids were centrifuged and washed several times with EtOH. The NPs were redispersed in $1.0 \mathrm{~mL}$ absolute EtOH to obtain $0.2 \mathrm{mg} \mathrm{mL}{ }^{-1} \mathrm{SiO}_{2} @ \mathrm{Au} @ A g$ NPs modified with ATP (based on $\mathrm{SiO}_{2}$ ).

\subsection{SERS measurement of the $\mathrm{SiO}_{2} @ \mathrm{Au} @ A \mathrm{Ag}$ NPs}

$\mathrm{SiO}_{2} @ \mathrm{Au} @ A g$ NPs modified with ATP were spread on a glass slide by the drop-casting method and SERS signals were measured using a confocal micro-Raman system (LabRam 300, JY-Horiba, Tokyo, Japan) equipped with an optical microscope (BX41, Olympus, Tokyo, Japan). The SERS signals were collected in a back-scattering geometry using $\times 10$ objective lens $(0.90 \mathrm{NA}$, Olympus) and a spectrometer equipped with a thermoelectric cooled CCD detector. A $532 \mathrm{~nm}$ diode-pumped solid-state laser (CL532-100-S; Crystalaser) was used as the photo-excitation source, with $10 \mathrm{~mW}$ laser power at the sample. The strong Rayleigh scattered light was rejected using a long-pass filter. Selected sites were measured randomly, and all SERS spectra were integrated for $5 \mathrm{~s}$. The spot size of the laser beam was about $2 \mu \mathrm{m}$.

\section{7 $\quad E$-Field distribution calculation using the DDA calculation for identifying SERS enhancement}

The $E$-fields of Au and the Au@Ag NPs dimer were calculated using the DDA package (DDSCAT 7.1) to investigate the SERS enhancement of $\mathrm{SiO}_{2} @ \mathrm{Au} @ \mathrm{Ag} \mathrm{NPs}^{50}$ The diameter of an $\mathrm{Au} \mathrm{NP}$ was $3 \mathrm{~nm}$ based on the TEM size analysis of $\mathrm{SiO}_{2} @ A u$ NPs, and $\mathrm{Ag}$ shell thickness on the Au@Ag NPs was 2.5-10 nm. The interdipole distance was $0.25 \mathrm{~nm}$, and the dielectric constant was obtained from Palik. ${ }^{51}$ The medium was set as vacuum with a refractive index of $1.00+0 \mathrm{i}$. Wavelength was $532 \mathrm{~nm}$.

\section{Conclusions}

In conclusion, we have successfully developed highly sensitive SERS probes based on the $\mathrm{SiO}_{2} @ A u @ A g$ NPs using the Au NPs mediated $\mathrm{Ag}$ growth method. The SERS activities of the $\mathrm{SiO}_{2}$ @$\mathrm{Au} @ \mathrm{Ag}$ NPs were tuned by adjusting the $\mathrm{AgNO}_{3}$ concentration, resulting in the formation of nanogaps between two $\mathrm{Ag}$ NPs on the surface of the $\mathrm{SiO}_{2} @ A u @ A g$ NP. Strong Raman signals obtained were originated from a highly enhanced $E$-field at the nanogaps ( $<1 \mathrm{~nm}$ ) between two Ag NPs of the $\mathrm{SiO}_{2} @ \mathrm{Au} @ \mathrm{Ag} \mathrm{NPs}$. In addition, the $\mathrm{SiO}_{2} @ A u @ A g$ NPs exhibited LOD of $2.4 \mathrm{nM}$ for ATP. Moreover, Raman signals of ATP with $\mathrm{SiO}_{2} @ A u @ A g$ NPs showed a high reproducibility ( $\sim 14.2 \%$ of deviation) in batch-tobatch experiments. The $\mathrm{SiO}_{2} @ A u @ A g$ NPs can be utilized as a highly sensitive SERS substrate and used for detection of trace amounts of chemicals in various fields.

\section{Conflict of interest}

We have no conflicts of interest.

\section{Acknowledgements}

This work was supported by the Bio \& Medical Technology Development Program of the National Research Foundation (NRF) \& funded by the Korean government (MSIP \& MOHW) (2016-A423-0045). 


\section{References}

1 S. Schlücker, Angew. Chem., Int. Ed., 2014, 53, 4756-4795.

2 Y. Wang, B. Yan and L. Chen, Chem. Rev., 2013, 113, 13911428.

3 M. Culha, B. Cullum, N. Lavrik and C. K. Klutse, Nanotechnology, 2012, 2012, 15.

4 B.-H. Jun, G. Kim, S. Jeong, M. S. Noh, X.-H. Pham, H. Kang, M.-H. Cho, J.-H. Kim, Y.-S. Lee and D. H. Jeong, Bull. Korean Chem. Soc., 2015, 36, 963-978.

5 S. Nie and S. R. Emory, Science, 1997, 275, 1102-1106.

6 K. Sivashanmugan, J.-D. Liao, B. H. Liu, C.-K. Yao and S.-C. Luo, Sens. Actuators, B, 2015, 207, 430-436.

7 Y. Hu, J. Liao, D. Wang and G. Li, Anal. Chem., 2014, 86, 3955-3963.

8 M. R. Jones, K. D. Osberg, R. J. Macfarlane, M. R. Langille and C. A. Mirkin, Chem. Rev., 2011, 111, 3736-3827.

9 M. J. Banholzer, J. E. Millstone, L. Qin and C. A. Mirkin, Chem. Soc. Rev., 2008, 37, 885-897.

10 C. E. Talley, J. B. Jackson, C. Oubre, N. K. Grady, C. W. Hollars, S. M. Lane, T. R. Huser, P. Nordlander and N. J. Halas, Nano Lett., 2005, 5, 1569-1574.

11 J. B. Jackson and N. J. Halas, Proc. Natl. Acad. Sci. U. S. A., 2004, 101, 17930-17935.

12 J. B. Jackson and N. J. Halas, J. Phys. Chem. B, 2001, 105, 2743-2746.

13 W. R. Erwin, A. Coppola, H. F. Zarick, P. Arora, K. J. Miller and R. Bardhan, Nanoscale, 2014, 6, 12626-12634.

14 Q. Wu, C. Zhang and F. Li, Mater. Lett., 2005, 59, 3672-3677.

15 J.-H. Kim, W. W. Bryan and T. Randall Lee, Langmuir, 2008, 24, 11147-11152.

16 Z.-j. Jiang and C.-y. Liu, J. Phys. Chem. B, 2003, 107, 1241112415.

17 J. D. Driskell, R. J. Lipert and M. D. Porter, J. Phys. Chem. B, 2006, 110, 17444-17451.

18 L. O. Brown and S. K. Doorn, Langmuir, 2008, 24, 2277-2280.

19 X. Su, J. Zhang, L. Sun, T.-W. Koo, S. Chan, N. Sundararajan, M. Yamakawa and A. A. Berlin, Nano Lett., 2005, 5, 49-54.

20 B.-H. Jun, J.-H. Kim, H. Park, J.-S. Kim, K.-N. Yu, S.-M. Lee, H. Choi, S.-Y. Kwak, Y.-K. Kim, D. H. Jeong, M.-H. Cho and Y.-S. Lee, ACS Comb. Sci., 2007, 9, 237-244.

21 K. Kim, H. B. Lee, H. K. Park and K. S. Shin, J. Colloid Interface Sci., 2008, 318, 195-201.

22 J.-M. Li, W.-F. Ma, C. Wei, J. Guo, J. Hu and C.-C. Wang, J. Mater. Chem., 2011, 21, 5992-5998.

23 H. Kang, T. Kang, S. Kim, J.-H. Kim, B.-H. Jun, J. Chae, J. Park, D.-H. Jeong and Y.-S. Lee, J. Nanosci. Nanotechnol., 2011, 11, 579-583.

24 H. Kang, J. Yim, S. Jeong, J.-K. Yang, S. Kyeong, S.-J. Jeon, J. Kim, K. D. Eom, H. Lee, H.-I. Kim, D. H. Jeong, J.-H. Kim and Y.-S. Lee, ACS Appl. Mater. Interfaces, 2013, 5, 1280412810.

25 H. Kang, J.-K. Yang, M. S. Noh, A. Jo, S. Jeong, M. Lee, S. Lee, H. Chang, H. Lee, S.-J. Jeon, H.-I. Kim, M.-H. Cho, H.-Y. Lee, J.-H. Kim, D. H. Jeong and Y.-S. Lee, J. Mater. Chem. B, 2014, 2, 4415-4421.
26 H. Chang, H. Kang, J.-K. Yang, A. Jo, H.-Y. Lee, Y.-S. Lee and D. H. Jeong, ACS Appl. Mater. Interfaces, 2014, 6, 1185911863.

27 J.-K. Yang, H. Kang, H. Lee, A. Jo, S. Jeong, S.-J. Jeon, H.-I. Kim, H.-Y. Lee, D. H. Jeong, J.-H. Kim and Y.-S. Lee, ACS Appl. Mater. Interfaces, 2014, 6, 12541-12549.

28 J.-H. Kim, J.-S. Kim, H. Choi, S.-M. Lee, B.-H. Jun, K.-N. Yu, E. Kuk, Y.-K. Kim, D. H. Jeong, M.-H. Cho and Y.-S. Lee, Anal. Chem., 2006, 78, 6967-6973.

29 C. Wang, Y. Chen, T. Wang, Z. Ma and Z. Su, Adv. Funct. Mater., 2008, 18, 355-361.

30 J.-M. Li, W.-F. Ma, C. Wei, L.-J. You, J. Guo, J. Hu and C.-C. Wang, Langmuir, 2011, 27, 14539-14544.

31 K. Wang, X. Zhang, C. Niu and Y. Wang, ACS Appl. Mater. Interfaces, 2014, 6, 1272-1278.

32 M. Chen, Y. N. Kim, H. M. Lee, C. Li and S. O. Cho, J. Phys. Chem. C, 2008, 112, 8870-8874.

33 S. L. Westcott, S. J. Oldenburg, T. R. Lee and N. J. Halas, Langmuir, 1998, 14, 5396-5401.

34 J. Xue, C. Wang and Z. Ma, Mater. Chem. Phys., 2007, 105, 419-425.

35 B. Sadtler and A. Wei, Chem. Commun., 2002, 1604-1605, DOI: $10.1039 / \mathrm{b} 204760 \mathrm{~h}$.

36 H. Hiramatsu and F. E. Osterloh, Langmuir, 2003, 19, 70037011.

37 C. Graf, S. Dembski, A. Hofmann and E. Rühl, Langmuir, 2006, 22, 5604-5610.

38 L. Lu, H. Zhang, G. Sun, S. Xi, H. Wang, X. Li, X. Wang and B. Zhao, Langmuir, 2003, 19, 9490-9493.

39 S. Pang, T. Yang and L. He, TrAC, Trends Anal. Chem., 2016, 85, 73-82.

40 S. Lee, H. Chon, M. Lee, J. Choo, S. Y. Shin, Y. H. Lee, I. J. Rhyu, S. W. Son and C. H. Oh, Biosens. Bioelectron., 2009, 24, 2260-2263.

41 D. G. Duff, A. Baiker and P. P. Edwards, Langmuir, 1993, 9, 2301-2309.

42 D. A. Genov, A. K. Sarychev and V. M. Shalaev, J. Nonlinear Opt. Phys. Mater., 2003, 12, 419-440.

43 D.-F. Zhang, L.-Y. Niu, L. Jiang, P.-G. Yin, L.-D. Sun, H. Zhang, R. Zhang, L. Guo and C.-H. Yan, J. Phys. Chem. $C$, 2008, 112, 16011-16016.

44 N. G. Bastús, F. Merkoçi, J. Piella and V. Puntes, Chem. Mater., 2014, 26, 2836-2846.

45 A. Biswas, H. Eilers, F. Hidden, O. C. Aktas and C. V. S. Kiran, Appl. Phys. Lett., 2006, 88, 013103.

46 K. Kneipp, H. Kneipp and J. Kneipp, Acc. Chem. Res., 2006, 39, 443-450.

47 D. D. A. Grube, T. Kiely and L. Wu, U. S. Environ. Prot. Agency, 2011.

48 D.-K. Lim, K.-S. Jeon, H. M. Kim, J.-M. Nam and Y. D. Suh, Nat. Mater., 2010, 9, 60-67.

49 W. Stöber, A. Fink and E. Bohn, J. Colloid Interface Sci., 1968, 26, 62-69.

50 B. T. Draine and P. J. Flatau, J. Opt. Soc. Am. A, 1994, 11, 1491-1499.

51 E. D. Palik, Handbook of Optical Constants of Solids, Academic press, 1991. 